TAIWANESE JOURNAL OF MATHEMATICS

Vol. 15, No. 4, pp. 1677-1690, August 2011

This paper is available online at http://www.tjm.nsysu.edu.tw/

\title{
NON-CLASSICAL ORTHOGONALITY RELATIONS FOR CONTINUOUS $q$-JACOBI POLYNOMIALS
}

\author{
Samuel G. Moreno* and Esther M. Garcǐa-Caballero
}

\begin{abstract}
We consider the continuous $q$-Jacobi polynomials $\left\{P_{n}^{(\alpha, \beta)}(\cdot \mid q)\right\}_{n=0}^{\infty}$, extending the variable and the parameters beyond classical considerations. For those new allowed values of the parameters for which Favard's theorem fails to work, we construct inner products in which the presence of the AskeyWilson divided difference operator provides the $q$-Sobolev character of the non-standard orthogonality for the corresponding family.
\end{abstract}

\section{INTRODUCTION}

In this paper, $\mathbb{N}, \mathbb{N}_{0}, \mathbb{R}$, and $\mathbb{C}$ will stand, respectively, for the sets of positive integers, the nonnegative integers, the real number set and the set of complex numbers. $\mathbb{P}$ will denote the set of algebraic polynomials in one complex variable. The notations and terminologies concerning the $q$-techniques that we will adopt in the sequel are the customary ones. We refer the reader to [10] and [17] for further details. We shall always assume $0<q<1$. The so-called $q$-shifted factorial is defined, for $x \in \mathbb{R}$, by

$$
(x ; q)_{0}=1, \quad(x ; q)_{n+1}=\prod_{k=0}^{n}\left(1-x q^{k}\right), \quad n \geq 0, \quad(x ; q)_{\infty}=\prod_{k=0}^{\infty}\left(1-x q^{k}\right) .
$$

For $x_{1}, x_{2}, \ldots, x_{m} \in \mathbb{R}$, the $q$-multi-shifted factorial is defined by means of,

$$
\left(x_{1}, x_{2}, \ldots, x_{m} ; q\right)_{n}=\prod_{k=1}^{m}\left(x_{k} ; q\right)_{n} .
$$

For a non-negative integer $n$, and for $0 \leq k \leq n$, the $q$-binomial coefficient is

Received January 15, 2010, accepted April 6, 2010.

Communicated by H. M. Srivastava.

2000 Mathematics Subject Classification: 33D45, 42C05.

Key words and phrases: Continuous $q$-Jacobi polynomials, Non-standard orthogonality, Favard's theorem.

This work was partially supported by Junta de Andalucía, Research Group FQM 0178, and by Ministerio de Educacion y Ciencia, Project MTM2006-14590.

*Corresponding author. 


$$
\left[\begin{array}{l}
n \\
k
\end{array}\right]_{q}=\frac{(q ; q)_{n}}{(q ; q)_{k}(q ; q)_{n-k}}
$$

A basic hypergeometric (also, $q$-hypergeometric) series ${ }_{m} \phi_{n}$ is defined by

$$
\begin{aligned}
& { }_{m} \phi_{n}\left(\begin{array}{c}
a_{1}, \ldots, a_{m} \\
b_{1}, \ldots, b_{n}
\end{array} \mid q ; x\right) \\
= & \sum_{k=0}^{\infty} \frac{\left(a_{1} ; q\right)_{k} \cdots\left(a_{m} ; q\right)_{k}}{\left(b_{1} ; q\right)_{k} \cdots\left(b_{n} ; q\right)_{k}} \frac{(-1)^{(n-m+1) k}}{q^{(m-n-1) k(k-1) / 2}} \frac{x^{k}}{(q ; q)_{k}} .
\end{aligned}
$$

We recall that the Askey-Wilson operator $D_{q}: \mathbb{P} \rightarrow \mathbb{P}$ (see [7]) is defined for polynomials that can be viewed as symmetric Laurent polynomials $\widehat{p}$, defined by means of $\widehat{p}(z)=\sum_{k=-n}^{k=n} c_{k} z^{k}$, where $c_{k}=c_{-k}$, which are related to polynomials $p$ in $x=\left(z+z^{-1}\right) / 2$ by $p(x)=p\left(\frac{1}{2}\left(z+z^{-1}\right)\right)=\widehat{p}(z)$. With this in mind, the operator $D_{q}$ is defined by

$$
D_{q} p(x)=\frac{\delta_{q} \widehat{p}(z)}{\delta_{q} \widehat{x}(z)}, \quad p \in \mathbb{P},
$$

where $\widehat{x}$ stands for the symmetric Laurent polynomial defined by $\widehat{x}(z)=(z+$ $\left.z^{-1}\right) / 2$, and where for each function $f$ in the variable $z, \delta_{q} f(z)=f\left(q^{1 / 2} z\right)-$ $f\left(q^{-1 / 2} z\right)$. Therefore,

$$
D_{q} p(x)=\frac{2\left(\widehat{p}\left(q^{\frac{1}{2}} z\right)-\widehat{p}\left(q^{-\frac{1}{2}} z\right)\right)}{\left(q^{\frac{1}{2}}-q^{-\frac{1}{2}}\right)\left(z-z^{-1}\right)}, \quad p \in \mathbb{P} .
$$

If for each $z \in \mathbb{C} \backslash\{0\}$ we define $\theta=-i \log (z)$, then $x=\cos \theta$ and the formula for $D_{q} p(x)$ can be rewritten

$$
D_{q} p(x)=\frac{\widehat{p}\left(q^{\frac{1}{2}} e^{i \theta}\right)-\widehat{p}\left(q^{-\frac{1}{2}} e^{i \theta}\right)}{i\left(q^{\frac{1}{2}}-q^{-\frac{1}{2}}\right) \sin \theta}, \quad x, \theta \in \mathbb{C}, \quad x=\cos \theta, \quad p \in \mathbb{P} .
$$

In a previous contribution [13] we established orthogonality relations for the families of big and little $q$-Jacobi polynomials having parameters for which Favard's theorem does not work. In the present paper, which can be considered as a short follow-up of [13], we investigate the corresponding non-classical orthogonality relations for the other celebrated $q$-analogues of the very classical Jacobi polynomials, namely, the continuous $q$-Jacobi polynomials $\left\{P_{n}^{(\alpha, \beta)}(\cdot \mid q)\right\}_{n=0}^{\infty}$. The structure of the paper is the following:

(1) Section 2 is devoted to introducing some basic facts about continuous $q$ Jacobi polynomials.

(2) In Section 3 we will give the two key tools for the main result: The first one concerns with the iterative action of the Askey-Wilson divided difference operator 
on the continuous $q$-Jacobi polynomials; With respect to the second one, we will include some technical details (see Proof of Proposition 2) concerning the obtention of the roots of the continuous $q$-Jacobi polynomials with one parameter equals to a negative integer (i.e., when the tree term recurrence relation breaks down), which are totally different to the (direct but harder) ones used in [13] to factorize the big and little $q$-Jacobi polynomials, when their first parameter is equal to $q^{-N}(N \in \mathbb{N})$ and their last parameter does not belong to $\left\{q^{N-k}\right\}_{k \geq 2}$ (see [13, Propositions 2.3 and 4.3, Corollary 2.2] for details). Although this latter direct computation can be applied to factorize the continuous $q$-Jacobi polynomials $P_{n}^{(-N, \beta)}(x \mid q)$, with $n \geq N \in \mathbb{N}$ and $N-\beta-1 \notin \mathbb{N}$, which are one of the two families we are concerned with in this work, it has turned out to be inapplicable to the second one, that is, to the polynomials $P_{n}^{(\alpha,-N)}(x \mid q)$, with $n \geq N \in \mathbb{N}$ and $N-\alpha-1 \notin \mathbb{N}$. The technique developed in Section 3 has been successfully applied to solve this latter problem. Furthermore, it has been also applied in the cases of the continuous and the discrete $q$-ultraspherical polynomials [14]; moreover, it has led us to prove a result that can be paraphrased in the following way (we hope to discuss it elsewhere):

Theorem 1. All the polynomials in the Askey-scheme (after extending their parameters from its hypergeometric representation by simple algebraical manipulations) are orthogonal in the whole range of their parameters. Specifically, they become orthogonal for those cases for which the three term recurrence relation breaks down.

Particular cases of the above theorem can be found disperse in the literature: see $[15,18]$ for the Laguerre case, $[16,4,2,1]$ for the Jacobi case, $[3,8]$ for the Meixner case, [5, 6] for the case of symmetric Meixner-Pollaczek polynomials, [9] for (not necessarily symmetric) Meixner-Pollaczek polynomials with null parameter, [12] for all the pathological cases of Meixner-Pollaczek polynomials and, finally, [8] for classical families of polynomials which satisfy a discrete orthogonality with a finite number of masses (i.e., the Hahn, Racah, dual Hahn and Krawtchouk polynomials). We conjecture that Theorem 1 remains valid if "Askey-scheme" is changed by " $q$ Askey-scheme". The present paper, together with [13] and [14], can be considered as initial steps in proving our conjecture.

(iii) We give our main result in Section 4, in which we state the orthogonality of the continuous $q$-Jacobi polynomials for those outstanding cases in which the Favard's theorem does not work, that is, when their orthogonality, in principle, can not be ensured. The kinds of inner products that we use are in fact discretecontinuous bilinear forms, in which the discrete parts includes evaluations over the common roots of the considered polynomials, and the continuous parts has a weight with shifted parameters and acts over the product of the iterated AskeyWilson operator on the corresponding polynomials. The proof can be consider as an 
adapted modification of [11, Theorem 3]. For simplicity, we mimmic the structure of the proofs of Theorems 3.1,3.2, and 5.1 in [13], and to avoid duplicating similar calculations, we include only the specific details relative to the case of the continuous $q$-Jacobi polynomials considered here.

(iv) Finally, the particular case of the continuous $q$-Laguerre polynomials (which are a limit case of the continuous $q$-Jacobi polynomials), is considered in Section 5.

\section{On the Continuous $q$-Jacobi Polynomials}

In their impressive contribution [7], R. Askey and J. Wilson introduced, after giving a $q$-extension of the classical beta integral, a remarkable family of orthogonal polynomials (which carries four free complex parameters, other than $q$ ), with the property that almost all the classical orthogonal polynomials are placed on its boundary as special or as limit cases. The so-called Askey-Wilson polynomials $p_{n}(\cdot ; a, b, c, d \mid q)$ are defined, when normalized to be monic, by means of ([17, $3.1 .1,3.1 .5])$

$$
\begin{aligned}
& p_{n}(x ; a, b, c, d \mid q) \\
= & \frac{(a b, a c, a d ; q)_{n}}{2^{n} a^{n}\left(a b c d q^{n-1} ; q\right)_{n}}{ }_{4} \phi_{3}\left(\begin{array}{c}
q^{-n}, a b c d q^{n-1}, a e^{i \theta}, a e^{-i \theta} \\
a b, a c, a d
\end{array} \mid q ; q\right), n \in \mathbb{N}_{0},
\end{aligned}
$$

where the relation between the variables $x$ and $\theta$ must be understood as follows: first consider the complex variable $z=x+\left(x^{2}-1\right)^{1 / 2}$, where $\left(x^{2}-1\right)^{1 / 2}$ is chosen so that it lies in the same quadrant as $x$, except for $x \in[-1,1]$, along which the plane must be cut. Clearly, $z \neq 0$ and $z^{-1}=x-\left(x^{2}-1\right)^{1 / 2}$, so that $x=\left(z+z^{-1}\right) / 2$. Now define $\theta=-i \log (z)=-i \log \left(x+\left(x^{2}-1\right)^{1 / 2}\right)$, where $\log$ stands for the principal value of the complex logarithm function. Since $z=e^{i \theta}$, then we have

$$
x=\left(e^{i \theta}+e^{-i \theta}\right) / 2=\cos \theta .
$$

Although expressed as functions of $z=e^{i \theta}$, the Askey-Wilson polynomials depend on the complex variable $x$ due to the presence of the factor

$$
\begin{aligned}
& \left(a e^{i \theta} ; q\right)_{k}\left(a e^{-i \theta} ; q\right)_{k} \\
= & \prod_{i=1}^{k}\left(1-a e^{i \theta} q^{i-1}\right)\left(1-a e^{-i \theta} q^{i-1}\right)=\prod_{i=1}^{k}\left(1-a\left(e^{i \theta}+e^{-i \theta}\right) q^{i-1}+a^{2} q^{2(i-1)}\right) \\
= & \prod_{i=1}^{k}\left(1-2 a x q^{i-1}+a^{2} q^{2(i-1)}\right)
\end{aligned}
$$

in the $k$ th term $(1 \leq k \leq n)$ of the terminating basic hypergeometric series in (2.1).

Several special cases of the monic Askey-Wilson polynomials are $q$-analogues of the very classical monic Jacobi polynomials. The most important ones are 
(i) $P_{n}^{(\alpha, \beta)}(x \mid q)=p_{n}\left(x ; q^{\frac{1}{2}\left(\alpha+\frac{1}{2}\right)}, q^{\frac{1}{2}\left(\alpha+\frac{3}{2}\right)},-q^{\frac{1}{2}\left(\beta+\frac{1}{2}\right)},-q^{\frac{1}{2}\left(\beta+\frac{3}{2}\right)} \mid q\right)$,

(ii) $P_{n}^{(\alpha, \beta)}(x ; q)=p_{n}\left(x ; q^{\frac{1}{2}}, q^{\alpha+\frac{1}{2}},-q^{\beta+\frac{1}{2}},-q^{\frac{1}{2}} \mid q\right)$.

The first case is the choice of R. Askey and J. Wilson in [7], while the second one was introduced by M. Rahman in [19] (both cases were originally considered with a different normalization). These two specializations are not essentially different. In fact, they are related by a quadratic transformation, derived from a formula due to V.N. Singh [20], that reads

$$
P_{n}^{(\alpha, \beta)}\left(x \mid q^{2}\right)=P_{n}^{(\alpha, \beta)}(x ; q) .
$$

See also [17, 0.6.33 and p. 85], where a different normalization is considered. Both families, $\left\{P_{n}^{(\alpha, \beta)}(\cdot \mid q)\right\}_{n=0}^{\infty}$ and $\left\{P_{n}^{(\alpha, \beta)}(\cdot ; q)\right\}_{n=0}^{\infty}$, are called monic continuous $q$-Jacobi polynomials because each one of them becomes orthogonal with respect to an absolutely continuous measure.

In what follows, we will be concerned with the monic version of the continuous $q$-Jacobi polynomials just as originally introduced in [7].

For each $n \in \mathbb{N}_{0}$, the $n$th degree monic continuous $q$-Jacobi polynomial can be defined in terms of the basic hypergeometric series ${ }_{4} \phi_{3}$ by means of ([17, 3.10.1, $3.10 .4])$

$$
\begin{aligned}
& P_{n}^{(\alpha, \beta)}(x \mid q) \\
= & \frac{\left(q^{\alpha+1},-q^{\frac{1}{2}(\alpha+\beta+1)},-q^{\frac{1}{2}(\alpha+\beta+2)} ; q\right)_{n}}{2^{n} q^{\frac{n}{2}\left(\alpha+\frac{1}{2}\right)}\left(q^{\alpha+\beta+1+n} ; q\right)_{n}} \\
& \cdot 4 \phi_{3}\left(\begin{array}{c}
q^{-n}, q^{\alpha+\beta+1+n}, q^{\frac{1}{2}\left(\alpha+\frac{1}{2}\right)} e^{i \theta}, q^{\frac{1}{2}\left(\alpha+\frac{1}{2}\right)} e^{-i \theta} \\
q^{\alpha+1},-q^{\frac{1}{2}(\alpha+\beta+1)},-q^{\frac{1}{2}(\alpha+\beta+2)}
\end{array} \mid q ; q\right) \\
= & \sum_{k=0}^{n}\left((-1)^{k}\left[\begin{array}{c}
n \\
k
\end{array}\right]_{q} \frac{\left(q^{\alpha+1+k},-q^{\frac{1}{2}(\alpha+\beta+1)+k},-q^{\frac{1}{2}(\alpha+\beta+2)+k} ; q\right)_{n-k}}{\left(q^{\alpha+\beta+1+n+k} ; q\right)_{n-k}}\right. \\
& \left.\cdot 2^{-n} q^{\left(k(k+1)-2 k n-\left(\alpha+\frac{1}{2}\right) n\right) / 2}\left(q^{\frac{1}{2}\left(\alpha+\frac{1}{2}\right)} e^{i \theta}, q^{\frac{1}{2}\left(\alpha+\frac{1}{2}\right)} e^{-i \theta} ; q\right)_{k}\right), x=\cos \theta,
\end{aligned}
$$

where the second representation follows after applying a similar procedure as the one used in [13] for monic big and little $q$-Jacobi polynomials. We note that the explicit representation (2.2) is meaningful for those values of the parameters $\alpha$ and $\beta$ such that $-(\alpha+\beta+1) \notin \mathbb{N}$. In the classical setting, in which the parameters are restricted by the conditions $\alpha, \beta \geq-1 / 2$, the orthogonality relation is (see [17, $3.10 .2,3.10 .4])$ 


$$
\begin{aligned}
& \frac{1}{2 \pi} \int_{-1}^{1} P_{m}^{(\alpha, \beta)}(x \mid q) P_{n}^{(\alpha, \beta)}(x \mid q) \frac{w^{(\alpha, \beta)}(x \mid q)}{\sqrt{1-x^{2}}} d x \\
= & \frac{1}{2^{2 n}} \frac{\left(q^{\frac{1}{2}(\alpha+\beta+2)+n} ; q^{\frac{1}{2}}\right)_{\infty}}{\left(-q^{\frac{1}{2}(\alpha+\beta+1)+n} ; q^{\frac{1}{2}}\right)_{\infty}} \frac{1}{\left(q^{1+n}, q^{\alpha+1+n}, q^{\beta+1+n} ; q\right)_{\infty}} \\
& \frac{1}{\left(q^{\alpha+\beta+1+n} ; q\right)_{n}} \delta_{m n}, \quad m, n \in \mathbb{N}_{0},
\end{aligned}
$$

where

$$
w^{(\alpha, \beta)}(x \mid q)=\left|\frac{\left(e^{i \theta},-e^{i \theta} ; q^{\frac{1}{2}}\right)_{\infty}}{\left(q^{\frac{1}{2}\left(\alpha+\frac{1}{2}\right)} e^{i \theta},-q^{\frac{1}{2}\left(\beta+\frac{1}{2}\right)} e^{i \theta} ; q^{\frac{1}{2}}\right)_{\infty}}\right|^{2}, x=\cos \theta, \theta \in[0, \pi] .
$$

We recall that for real parameters $\alpha$ and $\beta$ such that $-(\alpha+\beta+1) \notin \mathbb{N}$, monic continuous $q$-Jacobi polynomials satisfy the three term recurrence relation (see [17, $3.10 .4,3.10 .3])$

$$
P_{n+1}^{(\alpha, \beta)}(x \mid q)=\left(x-A_{n}^{(\alpha, \beta \mid q)}\right) P_{n}^{(\alpha, \beta)}(x \mid q)-B_{n}^{(\alpha, \beta \mid q)} P_{n-1}^{(\alpha, \beta)}(x \mid q), n \geq 0,
$$

where

$$
\begin{gathered}
A_{n}^{(\alpha, \beta \mid q)}=\frac{1}{2}\left(q^{\frac{1}{2}\left(\alpha+\frac{1}{2}\right)}+q^{-\frac{1}{2}\left(\alpha+\frac{1}{2}\right)}\right. \\
-\frac{\left(1-q^{\alpha+1+n}\right)\left(1-q^{\alpha+\beta+1+n}\right)\left(1+q^{\frac{1}{2}(\alpha+\beta+1)+n}\right)\left(1+q^{\frac{1}{2}(\alpha+\beta+2)+n}\right)}{q^{\frac{1}{2}\left(\alpha+\frac{1}{2}\right)}\left(1-q^{\alpha+\beta+1+2 n}\right)\left(1-q^{\alpha+\beta+2+2 n}\right)} \\
\left.-\frac{q^{\frac{1}{2}\left(\alpha+\frac{1}{2}\right)}\left(1-q^{n}\right)\left(1-q^{\beta+n}\right)\left(1+q^{\frac{1}{2}(\alpha+\beta)+n}\right)\left(1+q^{\frac{1}{2}(\alpha+\beta+1)+n}\right)}{\left(1-q^{\alpha+\beta+2 n}\right)\left(1-q^{\alpha+\beta+1+2 n}\right)}\right), \\
B_{n}^{(\alpha, \beta \mid q)}=\frac{1}{4} \frac{\left(1-q^{n}\right)\left(1-q^{\alpha+n}\right)\left(1-q^{\beta+n}\right)\left(1-q^{\alpha+\beta+n}\right)}{\left(1-q^{\alpha+\beta-1+2 n}\right)\left(1-q^{\alpha+\beta+2 n}\right)^{2}\left(1-q^{\alpha+\beta+1+2 n}\right)} \\
\left(\left(1+q^{\frac{1}{2}(\alpha+\beta-1)+n}\right)\left(1+q^{\frac{1}{2}(\alpha+\beta)+n}\right)^{2}\left(1+q^{\frac{1}{2}(\alpha+\beta+1)+n}\right)\right) .
\end{gathered}
$$

Note that the restriction $\alpha+\beta \neq-1$ is not necessary in the previous result because when $n=1$, the factor $\left(1-q^{\alpha+\beta-1+2 n}\right)$ in the denominator of $B_{n}^{(\alpha, \beta \mid q)}$, simplifies with the factor $\left(1-q^{\alpha+\beta+n}\right)$ in its numerator. Moreover, being $N$ a positive integer, if either $\alpha=-N$ (being also $\beta \notin\{N-2, N-3, \ldots\}$ ), or $\beta=-N$ (being $\alpha \notin\{N-2, N-3, \ldots\}$ ), no orthogonality results can be deduced from Favard's theorem for the monic continuous $q$-Jacobi polynomials, due to the fact that $B_{N}^{(-N, \beta \mid q)}=B_{N}^{(\alpha,-N \mid q)}=0$. The main objective of this paper consists of giving orthogonality relations for these problematic values of the parameters for which the three term recurrence relation breaks down. 


\section{Two Steps Toward the Main Result}

Our first auxiliary result concerns with the very natural way in which the AskeyWilson divided difference operator $D_{q}$ acts on the monic continuous $q$-Jacobi polynomials.

Proposition 1. Let $\alpha$, $\beta$ be real numbers such that $-(\alpha+\beta+1) \notin \mathbb{N}$. For each nonnegative integer $n$,

$$
\begin{aligned}
& D_{q}^{k} P_{n}^{(\alpha, \beta)}(x \mid q) \\
= & \frac{\left(q^{n-k+1} ; q\right)_{k}}{(1-q)^{k}} q^{k(k+1-2 n) / 4} P_{n-k}^{(\alpha+k, \beta+k)}(x \mid q), \quad 0 \leq k \leq n+1 .
\end{aligned}
$$

Proof. Monic continuous $q$-Jacobi polynomials verify the forward shift relation (see $[17,3.10 .7,3.10 .4])$

$$
D_{q} P_{n}^{(\alpha, \beta)}(x \mid q)=\frac{1-q^{n}}{1-q} q^{(1-n) / 2} P_{n-1}^{(\alpha+1, \beta+1)}(x \mid q), \quad n \geq 0 .
$$

After $k$ iterations, we get the desired result.

The second auxiliary result deals with the obtention of $N$ common roots of the polynomials $P_{N+n}^{(-N, \beta)}(\cdot \mid q)$ (and also, $N$ common roots of the polynomials $P_{N+n}^{(\alpha,-N)}$ $(\cdot \mid q))$. For this purpose, we use a totally different technique that the one in [13].

Proposition 2. Fixed a positive integer $N$, let us define the $N$ distinct points

$$
x_{k}=\frac{1}{2}\left(q^{(2 N-4 k-1) / 4}+q^{-(2 N-4 k-1) / 4}\right), \quad 0 \leq k \leq N-1 .
$$

For all $n \geq N$ and for $k \in\{0,1, \ldots, N-1\}$,

$$
\begin{aligned}
P_{n}^{(-N, \beta)}\left(x_{k} \mid q\right) & =0, \quad N-\beta-1 \notin \mathbb{N}, \\
P_{n}^{(\alpha,-N)}\left(-x_{k} \mid q\right) & =0, \quad N-\alpha-1 \notin \mathbb{N} .
\end{aligned}
$$

Proof. The first step in the proof consists of proving that the following factorizations hold for each $n \geq N$ :

$$
\begin{aligned}
P_{n}^{(-N, \beta)}(x \mid q) & =P_{N}^{(-N, \beta)}(x \mid q) P_{n-N}^{(N, \beta)}(x \mid q) \\
& =(-1)^{N} 2^{-N} q^{\frac{N}{4}}\left(q^{\frac{1}{2}\left(\frac{1}{2}-N\right)} e^{i \theta}, q^{\frac{1}{2}\left(\frac{1}{2}-N\right)} e^{-i \theta} ; q\right)_{N} P_{n-N}^{(N, \beta)}(x \mid q),
\end{aligned}
$$

where $N-\beta-1 \notin \mathbb{N}$, and 


$$
\begin{aligned}
P_{n}^{(\alpha,-N)}(x \mid q) & =P_{N}^{(\alpha,-N)}(x \mid q) P_{n-N}^{(\alpha, N)}(x \mid q) \\
& =2^{-N} q^{\frac{N}{4}}\left(-q^{\frac{1}{2}\left(\frac{1}{2}-N\right)} e^{i \theta},-q^{\frac{1}{2}\left(\frac{1}{2}-N\right)} e^{-i \theta} ; q\right)_{N} P_{n-N}^{(\alpha, N)}(x \mid q),
\end{aligned}
$$

where $N-\alpha-1 \notin \mathbb{N}$. For this purpose, let us recall that monic continuous $q$-Jacobi polynomials, with parameters $\alpha$ and $\beta$, fulfill the three term recurrence relation (2.5). A direct computation on the coefficients $A_{n}^{(\alpha, \beta \mid q)}$ and $B_{n}^{(\alpha, \beta \mid q)}$ given by (2.6) and (2.7) shows that for each $k \geq 0$

$$
\begin{aligned}
& A_{N+k}^{(-N, \beta \mid q)}=A_{k}^{(N, \beta \mid q)}, \\
& B_{N+k}^{(-N, \beta \mid q)}=B_{k}^{(N, \beta \mid q) .}
\end{aligned}
$$

Now we use the principle of strong induction:

(i) For $n=N$ we trivially get that $P_{N}^{(-N, \beta)}(x \mid q)=P_{N}^{(-N, \beta)}(x \mid q) P_{0}^{(N, \beta)}(x \mid q)$.

(ii) Now suppose that for all $k$ such that $N \leq k \leq n$ we have $P_{k}^{(-N, \beta)}(x \mid q)=$ $P_{N}^{(-N, \beta)}(x \mid q) P_{k-N}^{(N, \beta)}(x \mid q)$. Using (2.5) with the choice $\alpha=-N$, using also (3.6) and (3.7), and taking into account the induction hypothesis, the expression for $P_{n+1}^{(-N, \beta)}(x \mid q)$ becomes

$$
\begin{aligned}
& P_{n+1}^{(-N, \beta)}(x \mid q) \\
= & \left(x-A_{n}^{(-N, \beta \mid q)}\right) P_{n}^{(-N, \beta)}(x \mid q)-B_{n}^{(-N, \beta \mid q)} P_{n-1}^{(-N, \beta)}(x \mid q) \\
= & P_{N}^{(-N, \beta)}(x \mid q)\left(\left(x-A_{n}^{(-N, \beta \mid q)}\right) P_{n-N}^{(N, \beta)}(x \mid q)-B_{n}^{(-N, \beta \mid q)} P_{n-1-N}^{(N, \beta)}(x \mid q)\right) \\
= & P_{N}^{(-N, \beta)}(x \mid q)\left(\left(x-A_{n-N}^{(N, \beta \mid q)}\right) P_{n-N}^{(N, \beta)}(x \mid q)-B_{n-N}^{(N, \beta \mid q)} P_{n-N-1}^{(N, \beta)}(x \mid q)\right) \\
= & P_{N}^{(-N, \beta)}(x \mid q) P_{n+1-N}^{(N, \beta)}(x \mid q) .
\end{aligned}
$$

Note that the above computation is valid for $n=N$, because although the induction hypothesis can not be used for $P_{N-1}^{(-N, \beta)}(x \mid q)$, the corresponding "correction" comes from the fact that $B_{N}^{(-N, \beta \mid q)}=P_{-1}^{(N, \beta)}(x \mid q)=0$. So we have proved the first equality of (3.4). The fact that

$$
P_{N}^{(-N, \beta)}(x \mid q)=(-1)^{N} 2^{-N} q^{\frac{N}{4}}\left(q^{\frac{1}{2}\left(\frac{1}{2}-N\right)} e^{i \theta}, q^{\frac{1}{2}\left(\frac{1}{2}-N\right)} e^{-i \theta} ; q\right)_{N}
$$

is straightforward from (2.2).

The first part of the factorization (3.5) can be derived by slight modifications of the argument above. To state the second part, first note that with the choice $\beta=-N$ and $n=N$ in the first equality in (2.2), we obtain 


$$
\begin{aligned}
P_{N}^{(\alpha,-N)}(x \mid q)= & \frac{\left(-q^{\frac{1}{2}(\alpha+1-N)},-q^{\frac{1}{2}(\alpha+2-N)} ; q\right)_{N}}{2^{N} q^{\frac{N}{2}\left(\alpha+\frac{1}{2}\right)}} \\
& \cdot 3 \phi_{2}\left(\begin{array}{c}
q^{-N}, q^{\frac{1}{2}\left(\alpha+\frac{1}{2}\right)} e^{i \theta}, q^{\frac{1}{2}\left(\alpha+\frac{1}{2}\right)} e^{-i \theta} \\
-q^{\frac{1}{2}(\alpha+1-N)},-q^{\frac{1}{2}(\alpha+2-N)}
\end{array} \mid q ; q\right) .
\end{aligned}
$$

Using the $q$-analogue of the Pfaff-Saalschütz summation formula ([17, 0.5.11])

$$
{ }_{3} \phi_{2}\left(\begin{array}{c}
q^{-n}, a, b \\
c, a b c^{-1} q^{1-n}
\end{array} \mid q ; q\right)=\frac{\left(a^{-1} c, b^{-1} c ; q\right)_{n}}{\left(c, a^{-1} b^{-1} c ; q\right)_{n}}, \quad n \in \mathbb{N}_{0},
$$

with $a=q^{\frac{1}{2}\left(\alpha+\frac{1}{2}\right)} e^{i \theta}, b=q^{\frac{1}{2}\left(\alpha+\frac{1}{2}\right)} e^{-i \theta}$, and $c=-q^{\frac{1}{2}(\alpha+1-N)}$, the expression above transforms to

$$
P_{N}^{(\alpha,-N)}(x \mid q)=\frac{\left(-q^{\frac{1}{2}(\alpha+2-N)} ; q\right)_{N}}{2^{N} q^{\frac{N}{2}\left(\alpha+\frac{1}{2}\right)}\left(-q^{-\frac{1}{2}(\alpha+N)} ; q\right)_{N}}\left(-q^{\frac{1}{2}\left(\frac{1}{2}-N\right)} e^{i \theta},-q^{\frac{1}{2}\left(\frac{1}{2}-N\right)} e^{-i \theta} ; q\right)_{N} .
$$

We conclude this part by noting that $\left(-q^{\frac{1}{2}(\alpha+2-N)} ; q\right)_{N} /\left(q^{\frac{N}{2}\left(\alpha+\frac{1}{2}\right)}\left(-q^{-\frac{1}{2}(\alpha+N)} ; q\right)_{N}\right)$ $=q^{N / 4}$.

The second (and last) step in the proof consist of proving (3.2) and (3.3). For this aim, we take into account, as proved above, that for all $n \geq N, P_{n}^{(-N, \beta)}(x \mid q)$ is a multiple of

$$
\begin{aligned}
\left(q^{\frac{1}{2}\left(\frac{1}{2}-N\right)} e^{i \theta}, q^{\frac{1}{2}\left(\frac{1}{2}-N\right)} e^{-i \theta} ; q\right)_{N} & =\prod_{k=0}^{N-1}\left(1-q^{\frac{1}{2}\left(\frac{1}{2}-N+2 k\right)} e^{i \theta}\right)\left(1-q^{\frac{1}{2}\left(\frac{1}{2}-N+2 k\right)} e^{-i \theta}\right) \\
& =\prod_{k=0}^{N-1}\left(1-2 q^{\frac{1}{2}\left(\frac{1}{2}-N+2 k\right)} \cos \theta+q^{\left(\frac{1}{2}-N+2 k\right)}\right)
\end{aligned}
$$

Imposing that $\left(q^{\frac{1}{2}\left(\frac{1}{2}-N\right)} e^{i \theta}, q^{\frac{1}{2}\left(\frac{1}{2}-N\right)} e^{-i \theta} ; q\right)_{N}$ vanishes, we get the roots (of $P_{n}^{(-N, \beta)}(\cdot \mid q)$, with $\left.n \geq N\right)$

$x_{k}=\cos \theta_{k}=\frac{1+q^{\left(\frac{1}{2}-N+2 k\right)}}{2 q^{\frac{1}{2}\left(\frac{1}{2}-N+2 k\right)}}=\frac{1}{2}\left(q^{(2 N-4 k-1) / 4}+q^{-(2 N-4 k-1) / 4}\right), 0 \leq k \leq N-1$.

To verify that the $-x_{k}$ 's are roots of $P_{n}^{(\alpha,-N)}(\cdot \mid q)$ for $n \geq N$, we use a similar argument.

A final detail: The fact that the points $x_{k}$ are distinct is a consequence that for $a>2$, the equation $a=x+x^{-1}$ has two roots $x_{1}, x_{2}$, related by $x_{1} x_{2}=$ 1. Therefore, the condition $x_{k_{1}}=x_{k_{2}}$ for $k_{1} \neq k_{2}$ should imply, either $0=$ $2 N-4 k_{1}-1+2 N-4 k_{2}-1=4\left(N-k_{1}-k_{2}\right)-2$, which is not possible, or $0=2 N-4 k_{1}-1-\left(2 N-4 k_{2}-1\right)=4\left(k_{2}-k_{1}\right)$, which implies $k_{1}=k_{2}$, a contradiction. 


\section{4. $D_{q}$-Sobolev Orthogonality of Continuous $q$-Jacobi Polynomials}

Now we are in position to state the main result of this paper. We will give orthogonality results for the families of continuous $q$-Jacobi polynomials with allowed parameters $\alpha, \beta$ such that the three term recurrence relation breaks down, i.e., when $\alpha=-N_{1}$ or $\beta=-N_{2}$, being $N_{1}, N_{2} \in \mathbb{N}$ and $\alpha+\beta \neq-2,-3, \ldots$. This will complete the treatment of this kind of orthogonality results for the most celebrated $q$-analogues of the very classical $q$-Jacobi polynomials.

For our aim we will apply the technique of our previous result [11, Theorem 3], using here the Askey-Wilson operator $D_{q}$ instead of the derivative one. The defined inner products will be called of type $D_{q}$-Sobolev because of the presence of the Askey-Wilson divided difference operator in their definitions.

Theorem 2. Fixed a positive integer $N$, let us define the $N$ distinct points

$$
x_{k}=\frac{1}{2}\left(q^{(2 N-4 k-1) / 4}+q^{-(2 N-4 k-1) / 4}\right), \quad 0 \leq k \leq N-1 .
$$

(i) For each $\beta \geq-N-1 / 2$ such that $\beta \notin\{-N,-N+1, \ldots, N-3, N-2\}$, there exists a symmetric and positive definite matrix $A_{1}$ of order $N$ such that the family of monic continuous q-Jacobi polynomials $\left\{P_{n}^{(-N, \beta)}(\cdot \mid q)\right\}_{n=0}^{\infty}$ is orthogonal with respect to the inner product $(\cdot, \cdot)_{1}^{\left(N ; A_{1} ; \beta \mid q\right)}$ defined by

$$
\begin{aligned}
& \left(p_{1}, p_{2}\right)_{1}^{\left(N ; A_{1} ; \beta \mid q\right)}=\left(\left(p_{1}\left(x_{k}\right)\right)_{k=0}^{N-1}\right) A_{1}\left(\left(p_{2}\left(x_{k}\right)\right)_{k=0}^{N-1}\right)^{t} \\
& \quad+\frac{1}{2 \pi} \int_{-1}^{1}\left(D_{q}^{N} p_{1}(x)\right)\left(D_{q}^{N} p_{2}(x)\right) \frac{w^{(0, \beta+N)}(x \mid q)}{\sqrt{1-x^{2}}} d x, \quad p_{1}, p_{2} \in \mathbb{P} .
\end{aligned}
$$

(ii) For each $\alpha \geq-N-1 / 2$ such that $\alpha \notin\{-N,-N+1, \ldots, N-3, N-2\}$, there exists a symmetric and positive definite matrix $A_{2}$ of order $N$ such that the family of monic continuous q-Jacobi polynomials $\left\{P_{n}^{(\alpha,-N)}(\cdot \mid q)\right\}_{n=0}^{\infty}$ is orthogonal with respect to the inner product $(\cdot, \cdot)_{2}^{\left(N ; A_{2} ; \alpha \mid q\right)}$ defined by

$$
\begin{aligned}
& \left(p_{1}, p_{2}\right)_{2}^{\left(N ; A_{2} ; \alpha \mid q\right)}=\left(\left(p_{1}\left(-x_{k}\right)\right)_{k=0}^{N-1}\right) A_{2}\left(\left(p_{2}\left(-x_{k}\right)\right)_{k=0}^{N-1}\right)^{t} \\
& \quad+\frac{1}{2 \pi} \int_{-1}^{1}\left(D_{q}^{N} p_{1}(x)\right)\left(D_{q}^{N} p_{2}(x)\right) \frac{w^{(\alpha+N, 0)}(x \mid q)}{\sqrt{1-x^{2}}} d x, \quad p_{1}, p_{2} \in \mathbb{P}
\end{aligned}
$$

In both cases, $w^{(0, \beta+N)}(\cdot \mid q)$ and $w^{(\alpha+N, 0)}(\cdot \mid q)$ can be computed by formula (2.4).

Proof. We can proceed, step by step, as in the theorems in Sections 3 and 5 in [13], adjusting the obvious modifications imposed by the cases we are studying here. So, maintaining the style of the current paper, we avoid duplicating similar calculations to the ones in [13]. Just some details: 
(i) For $i=1,2$, matrix $A_{i}$ equals $C_{i}^{-1} D^{2}\left(C_{i}^{-1}\right)^{t}$, where

$$
\begin{aligned}
C_{1} & =\left(P_{j}^{(-N, \beta)}\left(x_{k} \mid q\right)\right)_{j, k=0}^{N-1}, \\
C_{2} & =\left(P_{j}^{(\alpha,-N)}\left(-x_{k} \mid q\right)\right)_{j, k=0}^{N-1},
\end{aligned}
$$

and $D=\left(\kappa_{j} \delta_{j k}\right)_{j, k=0}^{N-1}$ is an arbitrary nonsingular diagonal matrix of order $N$, being $\kappa_{n}^{2}(0 \leq n \leq N-1)$ equals the square of the norm of the corresponding $n$-th continuous $q$-Jacobi polynomial.

(ii) When $n \geq N$, we get

$$
\begin{aligned}
& \left(P_{n}^{(-N, \beta)}(\cdot \mid q), P_{n}^{(-N, \beta)}(\cdot \mid q)\right)_{1}^{\left(N ; A_{1} ; \beta \mid q\right)} \\
= & \frac{q^{N(1+N-2 n) / 2}}{2^{2(n-N)}(1-q)^{2 N}\left(q^{1+n} ; q\right)_{\infty}^{2}} \kappa(n, N, \beta),
\end{aligned}
$$

and also

$$
\begin{aligned}
& \left(P_{n}^{(\alpha,-N)}(\cdot \mid q), P_{n}^{(\alpha,-N)}(\cdot \mid q)\right)_{2}^{\left(N ; A_{2} ; \alpha \mid q\right)} \\
= & \frac{q^{N(1+N-2 n) / 2}}{2^{2(n-N)}(1-q)^{2 N}\left(q^{1+n} ; q\right)_{\infty}^{2}} \kappa(n, N, \alpha),
\end{aligned}
$$

where $\kappa(n, N, \cdot)$ is defined by means of

$$
\kappa(n, N, \gamma)=\frac{\left(q^{\frac{1}{2}(\gamma+2-N)+n} ; q^{\frac{1}{2}}\right)_{\infty}}{\left(-q^{\frac{1}{2}(\gamma+1-N)+n} ; q^{\frac{1}{2}}\right)_{\infty}\left(q^{\gamma+1+n} ; q\right)_{\infty}\left(q^{\gamma+1+n} ; q\right)_{n-N}} .
$$

\section{Particular Case: The Continuous $q$-Laguerre Polynomials}

Monic continuous $q$-Laguerre polynomials $P_{n}^{(\alpha)}(\cdot \mid q)$ are obtained from monic continuous $q$-Jacobi polynomials $P_{n}^{(\alpha, \beta)}(\cdot \mid q)$ by taking the limit $\beta \rightarrow \infty$. Therefore, taking the limit $\beta \rightarrow \infty$ in all the results of Sections 2, 3 and 4 in which the parameter $\beta$ is free (i.e., wherever $\beta$ is not equal to a fixed $N \in \mathbb{N}$ ), we will obtain similar results to above ones, now for the family of continuous $q$-Laguerre polynomials. We will briefly summarize all these results, relating each of them with the corresponding formula in the preceding sections.

(i) Definition as a basic series (2.2)

Let $-\alpha \notin \mathbb{N}$. For each $n \in \mathbb{N}_{0}$ we define the $n$th degree monic continuous $q$-Laguerre polynomial $P_{n}^{(\alpha)}(\cdot \mid q)$ by

$$
P_{n}^{(\alpha)}(x \mid q)=\frac{\left(q^{\alpha+1} ; q\right)_{n}}{2^{n} q^{\frac{n}{2}\left(\alpha+\frac{1}{2}\right)}} 3 \phi_{2}\left(\begin{array}{c}
q^{-n}, q^{\frac{1}{2}\left(\alpha+\frac{1}{2}\right)} e^{i \theta}, q^{\frac{1}{2}\left(\alpha+\frac{1}{2}\right)} e^{-i \theta} \\
q^{\alpha+1}, 0
\end{array} \mid q ; q\right), x=\cos \theta .
$$


(ii) Orthogonality relation (2.3)

For $\alpha \geq-1 / 2$,

$$
\begin{aligned}
& \frac{1}{2 \pi} \int_{-1}^{1} P_{m}^{(\alpha)}(x \mid q) P_{n}^{(\alpha)}(x \mid q) \frac{w^{(\alpha)}(x \mid q)}{\sqrt{1-x^{2}}} d x \\
= & \frac{1}{2^{2 n}} \frac{1}{\left(q^{1+n}, q^{\alpha+1+n} ; q\right)} \delta_{m n}, \quad m, n \in \mathbb{N}_{0},
\end{aligned}
$$

where

$$
\text { (5.1) } \quad w^{(\alpha)}(x \mid q)=\left|\frac{\left(e^{i \theta},-e^{i \theta} ; q^{\frac{1}{2}}\right)_{\infty}}{\left(q^{\frac{1}{2}\left(\alpha+\frac{1}{2}\right)} e^{i \theta} ; q^{\frac{1}{2}}\right)_{\infty}}\right|^{2}, x=\cos \theta, \theta \in[0, \pi] .
$$

(iii) Generalized monic continuous q-Laguerre polynomials (2.2)

Let $\alpha \in \mathbb{R}$. For each $n \in \mathbb{N}_{0}$ we define the $n$th degree generalized monic continuous $q$-Laguerre polynomial $P_{n}^{(\alpha)}(\cdot \mid q)$ by

$$
\begin{aligned}
P_{n}^{(\alpha)}(x \mid q)= & \sum_{k=0}^{n}(-1)^{k}\left[\begin{array}{l}
n \\
k
\end{array}\right]_{q}\left(q^{\alpha+1+k} ; q\right)_{n-k} \\
& \cdot 2^{-n} q^{\left(k(k+1)-2 k n-\left(\alpha+\frac{1}{2}\right) n\right) / 2}\left(q^{\frac{1}{2}\left(\alpha+\frac{1}{2}\right)} e^{i \theta}, q^{\frac{1}{2}\left(\alpha+\frac{1}{2}\right)} e^{-i \theta} ; q\right)_{k},
\end{aligned}
$$

where $x=\cos \theta$.

(iv) Three term recurrence relation (2.5), (2.6), (2.7)

$$
P_{n+1}^{(\alpha)}(x \mid q)=\left(x-A_{n}^{(\alpha \mid q)}\right) P_{n}^{(\alpha)}(x \mid q)-B_{n}^{(\alpha \mid q)} P_{n-1}^{(\alpha)}(x \mid q), \quad n \geq 0,
$$

where $A_{n}^{(\alpha \mid q)}=q^{\frac{1}{2}\left(\alpha+\frac{1}{2}\right)+n}\left(1+q^{\frac{1}{2}}\right) / 2$ and $B_{n}^{(\alpha \mid q)}=\left(1-q^{n}\right)\left(1-q^{\alpha+n}\right) / 4$. When $-\alpha \in \mathbb{N}$, no orthogonality results can be deduced from Favard's theorem.

(v) Iterated forward shift operator (3.1)

For $n \in \mathbb{N}_{0}$ and $\alpha \in \mathbb{R}$,

$$
D_{q}^{k} P_{n}^{(\alpha)}(x \mid q)=\frac{\left(q^{n-k+1} ; q\right)_{k}}{(1-q)^{k}} q^{k(k+1-2 n) / 4} P_{n-k}^{(\alpha+k)}(x \mid q), \quad 0 \leq k \leq n+1 .
$$

(vi) Factorization (3.4)

For fixed $N \in \mathbb{N}$, and for all $n \geq N$,

$$
\begin{aligned}
P_{n}^{(-N)}(x \mid q) & =P_{N}^{(-N)}(x \mid q) P_{n-N}^{(N)}(x \mid q) \\
& =(-1)^{N} 2^{-N} q^{\frac{N}{4}}\left(q^{\frac{1}{2}\left(\frac{1}{2}-N\right)} e^{i \theta}, q^{\frac{1}{2}\left(\frac{1}{2}-N\right)} e^{-i \theta} ; q\right)_{N} P_{n-N}^{(N)}(x \mid q) .
\end{aligned}
$$


(vii) Evaluations on the lattice points (3.2)

For fixed $N \in \mathbb{N}$

$$
P_{n}^{(-N)}\left(x_{k} \mid q\right)=0, \quad n \geq N, \quad 0 \leq k \leq N-1 .
$$

where

$$
x_{k}=\frac{1}{2}\left(q^{(2 N-4 k-1) / 4}+q^{-(2 N-4 k-1) / 4}\right), \quad 0 \leq k \leq N-1 .
$$

(viii) $D_{q}$-Sobolev orthogonality (Theorem 2)

Theorem 3. For each positive integer $N$ there exists a symmetric and positive definite matrix $A$ of order $N$ such that the family of monic continuous $q$ Laguerre polynomials $\left\{P_{n}^{(-N)}(\cdot \mid q)\right\}_{n=0}^{\infty}$ is orthogonal with respect to the inner product $(\cdot, \cdot)_{1}^{(N ; A \mid q)}$ defined by

$$
\begin{aligned}
\left(p_{1}, p_{2}\right)_{1}^{(N ; A \mid q)}= & \left(\left(p_{1}\left(x_{k}\right)\right)_{k=0}^{N-1}\right) A\left(\left(p_{2}\left(x_{k}\right)\right)_{k=0}^{N-1}\right)^{t} \\
& +\frac{1}{2 \pi} \int_{-1}^{1}\left(D_{q}^{N} p_{1}(x)\right)\left(D_{q}^{N} p_{2}(x)\right) \frac{w^{(0)}(x \mid q)}{\sqrt{1-x^{2}}} d x, \quad p_{1}, p_{2} \in \mathbb{P}
\end{aligned}
$$

where the $x_{k}$ 's are given by (5.2) and $w^{(0)}(x \mid q)$ is the case $\alpha=0$ of (5.1).

\section{REFERENCES}

1. M. Alfaro, M. Álvarez de Morales and M. L. Rezola, Orthogonality of the Jacobi polynomials with negative integer parameters, J. Comput. Appl. Math., 145 (2002), 379-386.

2. M. Alfaro, T. E. Perez, M. A. Pinar and M. L. Rezola, Sobolev orthogonal polynomials: the discrete-continuous case, Meth. Appl. Anal., 6 (1999), 593-616.

3. M. Álvarez de Morales, T. E. Pérez, M. A. Piñar and A. Ronveaux, Non-standard orthogonality for Meixner polynomials, ETNA, Electron. Trans. Numer. Anal., 9 (1999), 1-25.

4. M. Álvarez de Morales, T. E. Perez and M. A. Piñar, Sobolev orthogonality for the Gegenbauer polynomials $\left\{C_{n}^{(-N+1 / 2)}\right\}_{n \geq 0}$, J. Comput. Appl. Math., 100 (1998), $111-120$

5. T. K. Araaya, The Meixner-Pollaczek polynomials and a system of orthogonal polynomials in a strip, J. Comput. Appl. Math., 170 (2004), 241-254.

6. T. K. Araaya, The symmetric Meixner-Pollaczek polynomials with real parameter, $J$. Math. Anal. Appl., 305 (2005), 411-423. 
7. R. Askey and J. Wilson, Some basic hypergeometric polynomials that generalize Jacobi polynomials, Memoirs Amer. Math. Soc., 319, Providence, Rhode Island, 1985.

8. R. S. Costas-Santos and J. F. Sánchez-Lara, Extensions of discrete classical orthogonal polynomials beyond the orthogonality, J. Comput. Appl. Math., 225 (2009), $440-451$

9. D. Dominici, Some remarks on a paper by L. Carlitz, J. Comput. Appl. Math., 198 (2007), 129-142.

10. G. Gasper and M. Rahman, Basic hypergeometric series, second ed., Cambridge Univ. Press, 2004.

11. Samuel G. Moreno and E. M. García-Caballero, Linear interpolation and Sobolev orthogonality, J. Approx. Theory, 161 (2008), 35-48.

12. Samuel G. Moreno and E. M. García-Caballero, The orthogonality of the MeixnerPollaczek polynomials $\left\{P_{n}^{\left(\frac{1-N}{2}\right)}(\cdot ; \phi)\right\}_{n=0}^{\infty}$ for positive integers $N$, submitted for publication.

13. Samuel G. Moreno and E. M. García-Caballero, Non-classical orthogonality relations for big and little $q$-Jacobi polynomials, J. Approx. Theory, 162 (2010), 303-322.

14. Samuel G. Moreno and E. M. Garcǐa-Caballero, New orthogonality relations for the continuous and the discrete $q$-ultraspherical polynomials, J. Math. Anal. Appl., 369 (2010), 386-399.

15. K. H. Kwon and L. L. Littlejohn, The orthogonality of the Laguerre polynomials $\left\{L_{n}^{-k}(x)\right\}$ for positive integers $k$, Ann. Numer. Math., 2 (1995), 289-303.

16. K. H. Kwon and L. L. Littlejohn, Sobolev orthogonal polynomials and second-order differential equations, Rocky Mountain J. Math., 28 (1998), 547-594.

17. R. Koekoek and R. F. Swarttouw, The Askey-scheme of hypergeometric orthogonal polynomials and its q-analogue, Technical Report 98-17, Delft University of Technology, 1998.

18. T. E. Pérez and M. A. Piñar, On Sobolev orthogonality for the generalized Laguerre polynomials, J. Approx. Theory, 86 (1996), 278-285.

19. M. Rahman, The linearization of the product of continuous $q$-Jacobi polynomials, Canad. J. Math., 33 (1981), 961-987.

20. V. N. Singh, The basic analogues of identities of the Cayley-Orr type, J. London Math. Soc., 34 (1959), 15-22.

Samuel G. Moreno and Esther M. García-Caballero

Departamento de Matemáticas

Universidad de Jaén

23071 Jaén

Spain

E-mail:samuel@ujaen.es

emgarcia@ujaen.es 